\title{
Pharmacology
}

\section{9th Symposium of the Austrian Pharmacological Society (APHAR)}

\author{
Vienna, November 21-22, 2003
}

\section{1 \\ Kynurenine Metabolism in Piglet Brain due to EMCV Infection \\ H. Baran a, b, M. Draxlera, b, B. Kepplinger ${ }^{\mathrm{f}}$, H. Kahlbacherc, N. Nowotny d, P. Schmidt ${ }^{\mathrm{e}}$, A. Urle, M. Schuh ${ }^{\mathrm{c}}$, G. Hofecker ${ }^{\mathrm{b}}$ a Pharmacology and Toxicology, b Physiology, c 2nd Med. Clinic, ${ }^{\mathrm{d} C l i n i c a l}$ Virology, e Pathology, Veterinary Medical

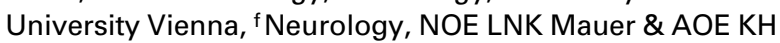 Amstetten, Austria}

Encephalomyocarditis virus (EMCV) mainly affects heart tissue and the central nervous system (CNS) and in piglets can cause sudden death. Kynurenic acid (KYNA) is an endogenous metabolite in the kynurenine pathway of tryptophan degradation and is an antagonist of EAA receptors. KYNA is synthesised from L-kynurenine by kynurenine aminotransferases I and II (KAT I and II). The aim of this study was to search activities of both enzymes in different brain regions at 4 days after EMCV (Belgian EMCV strain, B279/95) infection and in corresponding control (CO). On day 4 after infection the piglets were sacrificed and the brain regions were prepared for analyses. In EMCV-infected piglets we found moderate alterations of KAT I activity, whereas KAT II activity was increased in the medulla oblongata $(158 \%$ of $\mathrm{CO})$, piriform cortex $(147 \%$ of $\mathrm{CO})$, caudate nucleus ( $142 \%$ of $\mathrm{CO}$ ) and a moderate decrease was seen in the thalamus $(64 \%$ of $\mathrm{CO})$. Obtained data demonstrate affected KYNA metabolism in the CNS after EMCV infection.

Supported by Austrian Fond FWF P15371 to Halina Baran.

\section{2 \\ On the Fate of Skeletal Myoblasts in the Myocardium \\ S. Biryukova, H.C. Ott ${ }^{\mathrm{b}}$, R. Marksteiner ${ }^{\mathrm{a}}$, E. Margreiter ${ }^{\mathrm{a}}$, G. Laufer ${ }^{\mathrm{b}}, \mathrm{S}$. Hering ${ }^{\mathrm{c}}$ \\ a Department of Biochemical Pharmacology and ${ }^{b}$ Department of Cardiac Surgery, University of Innsbruck, c Department of \\ Pharmacology and Toxicology, University of Vienna, Austria}

The biophysical properties of skeletal muscle myoblasts transplanted into the infarcted myocardium of syngenic rats were analysed. For identification the myoblasts were permanently transfected with GFP and additionally labelled with the lipid dye PKH. The animals were sacrificed and $\operatorname{GFP}(+) / \mathrm{PKH}(+)$ myoblasts isolated for patch clamp studies. A sustained down-regulation of potassium, sodium and calcium voltage gated channels was observed. The fraction of cells with calcium channel currents declined within 24 hours from $68 \%$ to zero and the fraction of myoblasts with sodium currents within 1 week from $64 \%$ to zero. After 2 weeks of engraftment $30 \%$ of cells displayed potassium currents suggesting that down-regulation of this channel type was less pronounced. We observed an apparent switch in potassium channel expression. Before injection the myoblasts expressed predominantly transient outward potassium channels whereas in cells isolated from the myocardium almost exclusively rapid delayed rectifier channels with slow inactivation kinetics were observed. Ion channel down-regulation was reversible. The currents completely recovered between 1 and 6 weeks under cell culture conditions. The sustained down-regulation of ion channels and changes in potassium current kinetics suggest that the myocardial environment affects expression of voltage-gated ion channels of skeletal myoblasts. Our data indicate that during engraftment skeletal myoblasts enter a 'hibernating state' of reduced excitability.

S.B. and H.C.O. contributed equally to this work.

\section{KARGER (C) 2003 S. Karger AG, Basel


3

11-Dehydro-TXB 2 , a Stable Thromboxane Metabolite, Is a Full CRTH2 Agonist in Human Eosinophils and Basophils

E. Böhm ${ }^{\text {a, G.J. Sturm }}{ }^{\text {, }, ~ I . ~ W e i g l h o f e r a ~, ~ H . ~ S a n d i g b, ~}$ M. Shichijo c, A. McNammee d, J.E. Pease ${ }^{\mathrm{b}}$, B.A. Peskara, Á. Heinemann

a Department of Experimental and Clinical Pharmacology, Medical University Graz, Austria; bImperial College, South Kensington, London, UK; ' Bayer Yakuhin, Kyoto, Japan;

dGlaxoSmithKline, Stevenage, UK

Thromboxane (TX) $\mathrm{A}_{2}$, a cyclooxygenase-derived mediator involved in allergic responses, is rapidly converted in vivo to a stable metabolite, 11-dehydro- $\mathrm{TXB}_{2}$, which is considered to be biologically inactive. In this study we found that 11-dehydro- $\mathrm{TXB}_{2}$, but not the $\mathrm{TXA}_{2}$ analogue U46,619 or TXB 2 , activated eosinophils and basophils as assayed by flow cytometric shape change. 11-Dehydro-TXB was also chemotactic for eosinophils but did not induce, nor inhibit, platelet aggregation. CRTH2 is an important chemoattractant receptor expressed by eosinophils, basophils and Th2 lymphocytes, and prostaglandin $(\mathrm{PG}) \mathrm{D}_{2}$ has been shown to be its principal ligand. 11Dehydro- $\mathrm{TXB}_{2}$-induced calcium flux in eosinophils was mutually desensitized following stimulation with $\mathrm{PGD}_{2}$ but not other eosinophil chemoattractants. Shape change responses of eosinophils and basophils to 11-dehydro- $\mathrm{TXB}_{2}$ were inhibited by the thromboxane (TP)/CRTH2 receptor antagonist ramatroban, but not the selective $\mathrm{TP}$ antagonist SQ29,548. 11-Dehydro- $\mathrm{TXB}_{2}$ also induced the chemotaxis of $\mathrm{BaF} / 3$ cells transfected with hCRTH 2 but not naive $\mathrm{BaF} / 3$ cells. At a threshold concentration, 11-dehydro- $\mathrm{TXB}_{2}$ had no antagonistic effect on CRTH2-mediated responses as induced by $\mathrm{PGD}_{2}$. These data show that 11-dehydro-TXB ${ }_{2}$ is a full agonist of the $\mathrm{CRTH} 2$ receptor, and might hence be involved in allergic disease. Given its production in the allergic lung, antagonism of the CRTH2/ 11-dehydro- $\mathrm{TXB}_{2}$ axis may be of therapeutic relevance.

4

Recombinant Human Activated Protein C (rhAPC, Drotrecogin Alfa Activated) Has Minimal Effect on Markers of Coagulation, Fibrinolysis and Inflammation in Acute Human Endotoxemia

U. Derhaschnig a, b, R. Reiter ${ }^{\mathrm{a}}$, P. Knöb/c, M. Baumgartnera, P. Keen ${ }^{\mathrm{a}}$, B. Jilma ${ }^{\mathrm{a}, \mathrm{d}}$

Departments of a Clinical Pharmacology, ${ }^{b}$ Emergency

Medicine, ${ }^{\mathrm{c}}$ Haematology and ${ }^{\mathrm{d}}$ Pharmacology, University of

Vienna, Austria

Inflammatory and procoagulant host responses are closely related in sepsis. The protein $\mathrm{C}$ pathway serves as a regulatory pathway with anti-inflammatory and anticoagulant properties. Recently, recombinant human activated protein C (rhAPC) was shown to reduce mortality in severe sepsis. Nevertheless, the effects of rhAPC in humans are still ill defined. The infusion of low endotoxin doses into humans provides a standardized model to study inflammatory and hemostatic mechanisms. Thus, we investigated whether rhAPC acts as an anticoagulant or anti-inflammatory drug in human endotoxemia. 24 vol- unteers were randomized to receive either $24 \mu \mathrm{g} / \mathrm{kg} / \mathrm{h}$ rhAPC or placebo i.v. for $8 \mathrm{~h}$. LPS $(2 \mathrm{ng} / \mathrm{kg}$ ) was administered $2 \mathrm{~h}$ after starting the infusions. rhAPC decreased basal tissue factor (TF) mRNA expression, thrombin formation and action. In contrast, rhAPC did not significantly blunt LPS-induced thrombin generation. Consistently, rhAPC did not reduce LPS-induced levels of TF mRNA or D-dimer and had no effect on fibrinolytic activity or inflammation. Finally, endogenous APC formation was enhanced during endotoxemia and appeared to be associated with inflammation rather than thrombin formation. In conclusion, even low-grade endotoxemia induces significant protein $\mathrm{C}$ activation. Infusion of rhAPC decreases 'spontaneous' activation of coagulation but does not blunt LPS-induced, TFmediated coagulation in healthy volunteers, which is in contrast to a number of anticoagulants.

5

\section{Genomic Structure and Transcriptional} Regulation of Metabotropic Glutamate Receptors

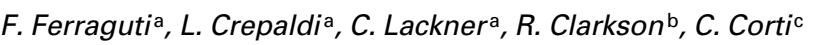

a Department of Pharmacology, University of Innsbruck, Austria; ${ }^{b}$ Department of Pathology, University of Cambridge, UK; ' Department of Biology, Glaxo SmithKline, Verona, Italy

Expression of metabotropic glutamate receptors (GRM) is mainly restricted to neural cells and undergoes dramatic changes in development as well as in neuropathological disorders or pharmacological treatments. This dynamic and cell-specific expression is dependent on each receptor distinctive genomic structure. We have elucidated the complete exon-intron structure of GRM1 and 5. Transcripts of these genes are generated by different alternatively spliced first exons. Transcription initiation of GRM5, identified by 5 '-RACE, takes place on three distinct exons: IA, IB and II. Their 5'-flanking regions were shown, using a luciferase reporter gene assay, to possess active promoter elements (CpG, TATA box, Oct-1) in neuroblastoma and glioma cells. Preferential luciferase activity was observed in neuroblastoma cells concomitant with differential DNA binding activity to responsive elements, such as CREB, Oct-1, C/EBP and Brn-2. Growth factors enhanced the activity of promoters IB and II in astroglioma cells and activated NF- $\mathrm{KB}$. These results suggest that alternative 5 -splicing and usage of multiple promoters contribute regulatory mechanisms for tissue- and context-specific expression.

\section{6 \\ EURO-MED-STAT Demonstrates Wide Variances in Availability, Prices and Utilisation of Medicines across Europe}

P. Folino-Gallo a, I. Rosian ${ }^{\mathrm{b}}, \mathrm{S}$. Vogler ${ }^{\mathrm{b}}$ et al. a Institute for Research on Population and Social Policies, Italian National Research Council, Rome, Italy; b ÖBIG

(Austrian Health Institute), Vienna, Austria

The aim of the EURO-MED-STAT project [Pharmacology 2002; 66:211-217] is to monitor prices, expenditure and utilisation of pharmaceuticals in Europe. Having collected the inventories of data 
sources on pharmaceutical prices, expenditure and utilisation in the EU member states and Norway and having assessed their reliability and comparability, there is evidence of wide variation in the availability of medicines across the surveyed countries, newer medicines tending to be more widely available. Taking the example of lipid lowering medicines, only five products are commonly available in all countries. This variation in availability and further differences (in pack size, dosages, and strength) hinders comparison. Substantial variation has also been observed concerning prices and utilisation of pharmaceuticals, as data collected on cardiovascular medicines in the European Union and Norway illustrate. For example, data of statins show high usage in Nordic countries, and least in Italy. Prescribing habits by the physicians, lifestyle factors (diet), morbidity patterns, and the use of lipid reducing medicines other than the statins are discussed as factors to explain these cross-country differences.

Supported by the European Commission, DG Health and Consumer Protection, Health Monitoring Programme.

\section{7}

\section{In vivo Characterization of the Cholecystokinin CCK $_{1}$ Receptor Agonist SR146131 in the Rat Pancreas}

\section{Groisman, T. Griesbacher}

Institute for Experimental and Clinical Pharmacology, University of Graz, Austria

Hyperstimulation of cholecystokinin $\mathrm{CCK}_{1}$ receptors by the $\mathrm{CCK}$ analogue caerulein (CRL) is the standard rat model for acute oedematous pancreatitis. However, CRL increases pancreatic blood flow via $\mathrm{CCK}_{2}$ receptors, which precludes conclusions about inflammation-related changes in tissue perfusion. SR146131 is a selective CCK $_{1}$ agonist [Bignon et al: J Pharmacol Exp Ther 1999;289:742751 and 752-761] and unlike previous compounds was believed to activate both high and low affinity $\mathrm{CCK}_{1}$ receptors. We have thus investigated whether SR146131 could be used as a new experimental model of pancreatitis. SR146131 stimulated enzyme secretion at doses about 10-fold higher than CRL, which was blocked by the $\mathrm{CCK}_{1}$ antagonist dexloxiglumide, but not by the $\mathrm{CCK}_{2}$ blocker itriglumide. However, in contrast to CRL the effects of SR146131 did not decline at supramaximal levels but remained at maximum values even at i.p. doses up to $1.8 \mu \mathrm{mol} / \mathrm{kg}$. While CRL induced pancreatitis at doses of $2.5 \mathrm{nmol} / \mathrm{kg}(2 \times$ at a $1 \mathrm{~h}$ interval $)$ and above, SR 146131 was devoid of such actions up to $1.8 \mu \mathrm{mol} / \mathrm{kg}$. In conclusion, SR146131 does not induce pancreatitis even at supramaximal dose levels and thus does not appear to activate low affinity $\mathrm{CCK}_{1}$ receptors in vivo.

Supported by ÖNB Jubilee Funds grant 9314.

\section{Depression-Like Behaviour Enhanced by Low Magnesium Diet: Investigation of Involved} Neuronal Substrates

\section{A. Hetzenauer, C. Sinner, H. Murck, N. Singewald Institute of Pharmacology and Toxicology, University of Innsbruck, Austria}

Magnesium deficiency is thought to be implicated in the pathophysiology of several diseases including stress-related disorders such as depression. We have recently shown that mice receiving a low magnesium diet display enhanced anxiety and depression-like behaviour in different behavioural tasks. The aim of the present study was to determine the neuroanatomical substrates involved in the behavioural effects in the forced swim test, using Fos immunoreactivity as a marker of neuronal activation. Mg-depleted $(\mathrm{MgD})$ mice ( $10 \%$ of the daily dietary requirement) displayed facilitated forced swimming-induced Fos expression in the central amygdala (CeA), which was reduced by chronic treatment with desipramine (DMI) and hypericum extract (HYP). Conclusion: Since the CeA is part of proposed depression circuits, enhanced neuronal activation in this brain area may be involved in the enhanced depression-related behavioural effects of $\mathrm{MgD}$ in mice. This conclusion is further supported by the attenuation of MgD-enhanced neuronal responsiveness in the amygdala by two different antidepressant treatments. The utility of $\mathrm{MgD}$ as a model to investigate neurobiological mechanisms of depression as well as of clinically active antidepressant drugs is suggested.

\section{9 \\ A Replicate Study Design for Testing Bioequivalence: A Case Study on Two Desmopressin Nasal Spray Preparations}

C. Joukhadara, b, B. Schenk ${ }^{\mathrm{a}}$, S.T. Kaehler ${ }^{\mathrm{c}}$, C.J. Kollenz ${ }^{\mathrm{c}}$, P. Bauerd, M. Müllera, H.-G. Eichler ${ }^{\mathrm{a}}$

a Department of Clinical Pharmacology, $b$ Institute of Pharmacology, University of Vienna, ' Medical Department, Gebro Pharma GmbH, Fieberbrunn, dInstitute of Medical

Statistics and Documentation, University of Vienna, Austria

Objective: To test bioequivalence (BE) between two different desmopressin nasal spray preparations. Due to the high variability of plasma pharmacokinetics (PK) of intranasally administered peptides like desmopressin, appropriate study designs are required to assess BE. Subjects and Methods: Thirty-two healthy male volunteers were enrolled in the study and were randomly assigned to receive the test and reference drug on two occasions in a 4-period 2-sequence crossover study design. Subjects received a single dose of $20 \mu \mathrm{g}(10 \mu \mathrm{g}$ per nostril) of desmopressin acetate per study day separated by washout periods of at least one week. Desmopressin blood concentrations were measured by using a validated radioimmunoassay method. Statistical analysis was initially performed using a complicated mixed analysis model testing for individual $\mathrm{BE}$ according to recommendations by the FDA. This approach, however, failed to converge with all defined main PK parameters and thus a traditional mixed analysis of variance analysis based on population averages was definitely used 
for testing BE between study drugs. The procedure of selecting an appropriate statistical analysis for a replicate study design was predefined in the study protocol. Results: The $90 \%$ confidence intervals (CI) were calculated for the area under the time-concentration curve (AUC), maximum concentration $\left(\mathrm{C}_{\max }\right)$ and the time to reach $\mathrm{C}_{\max }$ $\left(t_{\max }\right)$ of test/reference drug ratios for a BE range from 0.80 to 1.25 . The mean test/reference drug ratios were completely within the $90 \%$ CIs with values of 1.041 (CI: 0.892-1.216), 1.021 (CI: 0.913-1.140) and 1.068 (CI: 0.914-1.249) for $\mathrm{AUC}_{0-14 \mathrm{~h}}, \mathrm{C}_{\max }$ and $\mathrm{t}_{\max }$, respectively. Conclusion: The rate and the extent of intranasal desmopressin absorption are identical for both study preparations. Thus, the desmopressin test preparation met all $\mathrm{BE}$ criteria and thereby was proven bioequivalent with a marketed reference nasal desmopressin spray.

\section{0 \\ Penetration of Moxifloxacin into Healthy and Inflamed Subcutaneous Adipose Tissues in Humans}

C. Joukhadara , H. Stass b, U. Müller-Zellenberg a , E. Lacknera, F. Kovar a , E. Minarc, M. Müller ${ }^{\mathrm{a}}$

a Department of Clinical Pharmacology, University of Vienna, Austria; ${ }^{b}$ Bayer AG Pharma Res. Center, Inst. of Clinical Pharmacology, Wuppertal, Germany; ${ }^{c}$ Department of Internal Medicine II, Div. of Angiology, University of Vienna, Austria

Objective: The present study addressed the ability of moxifloxacin to penetrate into healthy and inflamed subcutaneous adipose tissue in twelve patients with soft tissue infection (STI). Patients and Methods: Penetration of moxifloxacin into the interstitial space fluid of healthy and inflamed subcutaneous adipose tissues was measured by use of in vivo microdialysis following a single intravenous dosage of $400 \mathrm{mg}$ in six diabetic and six non-diabetic patients suffering from STI. Results: For the entire study population the mean time-concentration profile of free moxifloxacin in plasma was identical to tissue time-concentration profiles $(p=n . s$.$) . For the diabetic$ subgroup mean moxifloxacin area under the time-concentration values from $0-8 \mathrm{~h}\left(\mathrm{AUC}_{0-8 \mathrm{~h}}\right)$ were $8.1 \pm 7.1 \mathrm{mg} \cdot \mathrm{h} / \mathrm{l}$ and $3.7 \pm 1.9$ $\mathrm{mg} \cdot \mathrm{h} / \mathrm{l}(\mathrm{p}=\mathrm{n} . \mathrm{s}$.) for healthy and inflamed adipose tissues, respectively. Mean $\mathrm{AUC}_{0-8} \mathrm{~h}$ inflamed tissue $/ \mathrm{AUC}_{0-8} \mathrm{~h}$ free plasma ratio was $0.5 \pm$ 0.4 for diabetics and $1.2 \pm 0.8$ for non-diabetic patients $(\mathrm{p}=\mathrm{n} . \mathrm{s})$. $\mathrm{AUC}_{0-24 \mathrm{~h} \text { free plasma }} / \mathrm{MIC}_{90}$ ratios were $>58$ and $121 \mathrm{~h}$ for Streptococcus species and methicillin-sensitive Staphylococcus aureus, respectively. Conclusion: Microbiologically effective concentrations of moxifloxacin are reached against clinically relevant bacterial strains in plasma, inflamed and healthy adipose tissue. Thus, tissue and plasma pharmacokinetics of moxifloxacin support its use for the treatment of STI in diabetic and non-diabetic patients.

\section{1 \\ Muscarinic Receptors Triggering Noradrenaline Release from Sympathetic Neurons}

S.G. Lechner, M. Mayer, S. Boehm

Department of Pharmacology, University of Vienna, Austria

$\mathrm{M}_{1}$ muscarinic receptors mediate excitation of sympathetic neurons through an inhibition of M-currents, but it is unclear whether this effect may trigger noradrenaline (NA) release. In cultures of rat SCG neurons, the muscarinic agonist oxotremorine $M(O x o M)$ inhibited M-currents $\left(\mathrm{EC}_{50} 1 \mu M\right)$ and induced NA release $\left(\mathrm{EC}_{50}\right.$ $10 \mu M)$. Both effects were not inhibited by the nicotinic antagonist mecamylamine. Inhibition of $\mathrm{G}_{\mathrm{i}}$-coupled pathways by pertussis toxin potentiated the release stimulating effect of OxoM $\left(\mathrm{EC}_{50} 3 \mu M\right)$. Pirenzepine antagonized M-current inhibition and the induction of release by OxoM with identical affinities, and both effects were abolished by MT7. Hence, both effects were mediated by $\mathrm{M}_{1}$ receptors. Retigabine abolished OxoM-induced, but not electrically evoked, release. Moreover, retigabine shifted the voltage dependence of Mcurrent activation to more negative potentials and hyperpolarized the neurons. OxoM depolarised neurons and elicited action potentials in 8 of 23 neurons; in the presence of retigabine, OxoM still caused equal depolarisations, but failed to trigger action potentials. Action potentials triggered by current injection were not affected by retigabine. Thus, activation of $\mathrm{M}_{1}$ receptors triggers transmitter release from sympathetic neurons through an inhibition of M-currents.

Supported by FWF P15797.

\section{2 \\ TRPC3 Function Is Dependent on the Structure of Cholesterol-Rich Microdomains

\author{
M. Lukasa, C. Roskera, H. Wolinskib, S.-D. Koh/wein b, \\ K. Groschner ${ }^{\mathrm{a}}$ \\ a Institute of Pharmacology and Toxicology, ${ }^{b}$ Institute of \\ Molecular Biology, Biochemistry and Microbiology, \\ Karl-Franzens-University of Graz, Austria
}

TRPC3 is a phospholipase C-regulated cation channel protein which appears to be targeted to specific microdomains of the plasma membrane. Fractionation of TRPC3-expressing HEK293 cells on a discontinuous sucrose gradient demonstrated that TRPC 3 is localized mainly in low-density fractions, which contain caveolin-1 (Cav1), representing cholesterol-rich microdomains classified as caveolae. Confocal imaging of cells expressing a YFP-TRPC3 fusion protein demonstrated that this protein was targeted to the plasma membrane, displaying a punctate pattern of distribution. Similarly, a CFP-Cav1 fusion protein was localized in punctate clusters in the plasma membrane. However, in cells coexpressing YFP-TRPC 3 and CFP-Cav1, the majority of YFP-TRPC3 clusters did not overlap with CFP-Cav1 fluorescence, and FRET between CFP and YFP was not detectable. These data indicate that TRPC 3 is mainly targeted to non-caveolar rafts, even though a clear functional link between Cav1 and TRPC3 was suggested from experiments measuring TRPC3mediated $\mathrm{Na}^{+}$as well as $\mathrm{Ca}^{2+}$ entry into HEK293 cells. Moreover, interventions which affect membrane cholesterol and the structure of 
raft domains were found to exert significant effects on TRPC 3 function. We suggest that albeit TRPC 3 is targeted to membrane microdomains different from caveolae, its function is strictly dependent on the integrity of cholesterol-rich rafts and may be governed by Cav1 via effects on membrane cholesterol distribution.

\section{3}

Familial Hemiplegic Migraine (FHM1) Mutants K1335E (KE), W1683R (WR) and V1695I (VI)

Alter $\mathrm{Ca}_{\mathrm{v}} 2.1 \mathrm{Ca}^{2+}$-Channel Function in a B-Subunit-Specific Manner

C. Müllnera, L.A.M. Broos ${ }^{\text {b }}$, R.R. Frants ${ }^{\text {b }}$, A.M.J.M. van den Maagdenberg ${ }^{\mathrm{b}}$, J. Striessniga

a Department of Pharmacology and Toxicology, University of Innsbruck, Austria; 'beiden University Medical Centre, Leiden, Netherlands

We studied the functional consequences of the new FHM1 mutations KE, WR and VI on human P/Q-type channels after coexpression in $X$. laevis oocytes $\left(\mathrm{Ca}_{\mathrm{v}} 2.1 \alpha 1+\alpha_{2} \delta+\right.$ various $\beta$-subunit isoforms). When coexpressed with $\beta 1$, barium inward current $\left(\mathrm{I}_{\mathrm{Ba}}\right)$ through all mutants was significantly shifted to more negative voltages. For VI this effect was absent with $\beta 3$ or $\beta 4$ coexpression. All mutants also significantly shifted the voltage for half-maximal channel inactivation to more negative potentials upon coexpression with $\beta 4$, but this effect was absent for VI coexpressed with $\beta 1$ or $\beta 3$. All mutants increased the accumulation of the channel in inactivation during trains of 15 100-ms depolarizations only with coexpressed $\beta 1$ and $\beta 3$, but not with $\beta 4$. $\beta$-subunit-specific changes on voltage-dependent current inactivation and recovery from inactivation were also observed in all three mutant channels. Our data support our previous hypothesis that enhanced channel activity during weak depolarizations is characteristic for FHM1 mutations. We also found that the mutant phenotype critically depends on $\beta$-subunit composition, suggesting that FHM1 mutant-induced changes of P/Q-type currents may vary between neurons expressing different types of $\beta$-subunits.

Supported by: EC (HPRN-CT-2000-00082), Austrian Science Fund (P-14541), the Austrian National Bank, and LUMC Stimulation Grant.

\section{4 \\ Statin-Induced Apoptosis in Human Skeletal Muscle Cells and Rhabdomyosarcoma Cells}

M. Werner, J. Sacher, L. Weigl, M. Hohenegger

Institute of Pharmacology, University of Vienna, Austria

Although HMG-CoA reductase inhibitors (statins) are successfully used in hypercholesterinaemia, in rare cases rhabdomyolysis is observed. The unknown reason of this side effect prompted us to investigate primary human skeletal muscle cells exposed to statins. Simvastatin induced apoptosis in a time- and concentration-dependent manner. The co-administration of mevalonic acid, the product of the HMG-CoA reductase, prevented statin-induced apoptosis. Delineation of the signalling pathway highlighted translocation of
Bax from the cytosol to the mitochondria as the crucial step in the activation of caspase 9 . In parallel, simvastatin activated an intracellular $\mathrm{Ca}^{2+}$ transient in fura-2 loaded human skeletal muscle cells which was even observed in the absence of extracellular $\mathrm{Ca}^{2+}$. Under these conditions calpain is activated and independent of the mitochondrial pathway of apoptosis. Making use of statin-induced apoptosis we could also show that human rhabdomyosarcoma cells (RD cells) respond in a similar manner. However calpain was not activated. Nevertheless, co-administration of the anthracyclin doxorubicin was additive to simvastatin in caspase 9 and 3 activation. Taken together these data show that statin-induced mitochondrial stress may explain rhabdomyolysis and a novel anti-cancer strategy.

\section{5 \\ Neuropeptide-Y $Y_{2}$ Receptors Inhibit the Afferent Input from the Acid-Challenged Stomach to the Mouse Brainstem}

\section{T. Wultsch a, R. Schicho a, H. Herzog b, G. Sperk c, P. Holzer ${ }^{\mathrm{a}}$ \\ a Department of Experimental and Clinical Pharmacology, University of Graz, Austria; b Garvan Institute of Medical \\ Research, Sydney, Australia; ${ }^{\mathrm{C} D e p a r t m e n t ~ o f ~ P h a r m a c o l o g y, ~}$ University of Innsbruck, Austria}

Background and Aims: Vagal afferents signal acid challenge of the rat gastric mucosa to the nucleus tractus solitarii (NTS) of the brainstem as visualized by expression of c-Fos. The aims of this study were (1) to study the vagal afferent signalling of gastric acid challenge in the mouse and (2) to explore whether neuropeptide-Y (NPY) participates in the processing of gastric afferent input to the NTS. The latter aim was addressed by the use of mice in which the NPY $Y_{2}$ receptor gene had been knocked out. Methods: The gastric mucosa of mice was exposed to different concentrations of acid $(0.15,0.25$ and $0.35 \mathrm{M} \mathrm{HCl})$ or saline $(0.01 \mathrm{ml} / \mathrm{g})$. Wild-type (FY2) and $\mathrm{Y}_{2}$ knockout (Y2KO) mice were treated with $0.25 \mathrm{M} \mathrm{HCl}$ intragastrically. Two hours later the brainstem was removed and activation of neurons in the NTS visualized by c-Fos immunohistochemistry. Results: Relative to saline, intragastric $\mathrm{HCl}(0.15-0.35 M)$ increased the total number of c-Fos protein-expressing cells in the mouse NTS in a concentration-dependent manner $(\mathrm{p}<0.01)$. The gastric acidevoked expression of c-Fos in the NTS of Y2KO mice was about $30 \%$ larger $(\mathrm{p}<0.05)$ than that in wildtype mice. Conclusions: Much as gastric acid exposure in the rat, gastric acid challenge in the mouse causes activation of neurons in the NTS, the central termination area of vagal afferents. NPY acting via $Y_{2}$ receptors appears to inhibit this stomach-brain communication process, either by a presynaptic site of action on vagal afferents or a postsynaptic site of action on NTS neurons.

Supported by the Jubilee Funds of the Austrian National Bank. 